\title{
La Fidelización del Cliente y Retención del Cliente: Tendencia que se Exige Hoy en Día
}

\section{Customer Loyalty and Customer Retention: \\ Trend Required Today}

\begin{abstract}
RESUMEN
El funcionamiento estable de la empresa depende directamente de su poder en el mercado y para ello es importante tener un alto grado de fidelización de los clientes y en caso estos no estén satisfechos con lo adquirido es importante que la empresa tenga la capacidad de revertirlo es decir retenerlo. Sin embargo, no existe una clara delimitación de ambos conceptos (fidelización y retención del cliente) y ese es el principal objetivo del artículo, pues es importante que las empresas tengan una idea clara sobre el significado de estas, con el fin que se apliquen políticas efectivas para que los clientes generen opiniones positivas sobre la empresa y lleguen a influenciar en la decisión de compra de nuevos individuos que no tienen claro a qué empresa acudir para satisfacer su necesidad y transmitir el sentimiento de seguridad que si no se llega a cumplir con las expectativas la empresa está dispuesta a corregir dicha situación.
\end{abstract}

Palabras claves: Cliente; fidelidad; retención y satisfacción.

\begin{abstract}
The stable operation of the company depends directly on its market power. For this it is important to have a high degree of customer loyalty and if they are not satisfied with what is acquired it is important that the company has the ability to reverse it. However, there is no clear delimitation of both concepts (customer loyalty and retention) and that is the main objective of the article, as it is important that companies have a clear idea about the meaning of these. With its main function that effective policies are applied so that customers generate positive opinions about the company and come to influence the purchase decision of new individuals who do not have a clear idea of the company, where they go to meet their need and transmit The feeling of security that fails to meet expectations and the company is willing to correct this situation.
\end{abstract}

Keywords: Customer; fidelity; retention and satisfaction.

\section{Sara Delfina Rosa Pierrend Hernández}

spierrendh@unmsm.edu.pe

Universidad Nacional Mayor de San Marcos, Facultad de Ciencias Administrativas. Lima, Perú

Presentado: 02/03/2020 - Aceptado: 19/05/2020 - Publicado: 20/10/2020

(C) Los autores. Este artículo es publicado por Gestión en el Tercer Milenio de la Facultad de Ciencias Administrativas de la Universidad Nacional Mayor de San Marcos. Este es un artículo de acceso abierto, distribuido bajo los términos de la licencia Creative Commons Atribucion - No Comercia_Compartir Igual 4.0 Internacional. (http://creativecommons.org/licenses/by-nc-sa/4.0/) que permite el uso no comercial, distribución y reproducción en cualquier medio, siempre que la obra original sea debidamente citada. 


\section{INTRODUCCIÓN}

El funcionamiento de la empresa a lo largo del tiempo depende directamente de su poder en el mercado, para ello es importante tener una gran parte del mercado interesado en adquirir los bienes o servicios que esta produzca. Por tanto, los conceptos de fidelización del cliente y la retención del cliente están en el foco del interés de todas las organizaciones, sin embargo, suele ser confuso el tratamiento de esta, tal vez por falta de una definición consensuada por académicos o por la similitud de los métodos que se utilizan para reforzar estos conceptos dentro de una empresa.

Para ello se explicarán los conceptos de cliente, y cómo es que un individuo pasa por un proceso para finalmente convertirse en cliente; lo que es la empresa, cómo esta tiene una actitud maximizadora; y finalmente, se explicará cómo es que la publicidad debe de dirigirse eficientemente para cumplir con los objetivos de la empresa.

Luego para determinar una definición de los conceptos de fidelización y retención del cliente, se tomarán los aportes de diferentes autores, con el fin de poder dar una delimitación general que también abarque la idea de los efectos que tiene la tecnología en cómo los individuos se comportan, ya que estos tienen preferencias cambiantes y es ahí donde radica el reto de las empresas por captar el interés de sus clientes por un largo tiempo. Se definirán ambos conceptos y explicará cómo es que se genera dentro de la empresa y que beneficios se tiene al reforzar la lealtad y las políticas para retener a los clientes.

Finalmente, se oficializará la delimitación de ambos conceptos como también los beneficios que estos dan a las empresas y se explicaran las diferencias de estos, explicando desde la premisa en que cada concepto genera y cuál es el nivel de satisfacción que tiene el cliente con los bienes o servicios adquiridos de la empresa.

\section{OBJETIVO DEL ARTÍCULO}

El presente artículo tiene como finalidad el brindar al lector una delimitación teórica y académica sobre los conceptos de fidelización del cliente y la retención del cliente. La delimitación de estos conceptos, tan usados para la implementación de ciertas políticas dentro de las organizaciones, estarán dentro de un contexto donde la información se traslada de manera rápida y los requerimientos de los clientes cambian constantemente influenciados por las redes sociales.

Además, se explicará el proceso de compra por el que pasa un individuo y se convierte en cliente de una empresa, enfatizando en las dos últimas fases de este los cuales son: decisión de compra y la conducta post-compra. Esto es importante porque se explica qué variables influyen en estas dos fases y cómo es que la empresa debería de actuar para minimizar el número de personas que desistan el consumir sus bienes o servicios.

Finalmente, se expondrán los beneficios que genera el reforzamiento de la fidelización de los clientes con la empresa o marca y la retención de estos para que se minimicen los costos que genera la pérdida de un cliente, pues es más rentable el obtener un cliente y que sea fiel a la empresa o marca en lugar de generar mayores gastos de marketing para obtener nuevos y transitorios. Por otro lado, se nombrarán ciertos métodos para medir la fidelización y retención de clientes.

\section{ARGUMENTACIÓN}

Tal como se determinó previamente, al momento de exponer los objetivos del artículo, se busca determinar una definición clara para los conceptos de fidelización del cliente y la retención del cliente de tal manera que se pueda diferenciar ambos conceptos, ya que al precisar dicha diferenciación se pueda tener idea de su aplicación y los beneficios que conlleva el reforzarlos dentro de las políticas que tengan las empresas sobre la relación que tendrán estas con sus clientes.

Por tal motivo, es importante iniciar explicando lo que se entiende por cliente, para ello se utiliza como punto de partida la definición de este concepto determinado por la Real Academia Española (RAE) la cual define el concepto cliente como "aquella persona que compra en un establecimiento o que adquiere algún servicio de un profesional o empresa". 
A partir de este concepto, debemos referirnos también al concepto de cliente no solo sobre la persona que se acerca y realiza una transacción en un determinado establecimiento, sino que el concepto que se propone va más allá, es algo más profundo dentro del contexto que se redacta en el presente artículo puesto que la tecnología ha provocado que el comercio sea mucho más rápido y variante al corto plazo. Con esto se generó que los medios en que se efectúen las transacciones cambien y se efectúen de diferentes formas, por lo que delimita como cliente, a la persona o empresa que genera un transacción o compromiso de compra futura con una empresa para adquirir un bien o servicio, siendo esta transacción de manera física o virtual.

Por otro lado, se aclara que el concepto de empresa que se toma para el resto del artículo como una entidad que utiliza el capital, materiales, talento humano y la tecnología para generar un producto o servicio que se pueda ofrecer dentro de un mercado determinado; estas entidades buscan generar más valor, para lo cual buscan maximizar sus beneficios tomando medidas para optimizar lo máximo posible los recursos utilizados, es decir, producir más beneficios utilizando los recursos necesario o mínimos para lograrlo.

Luego de tener delimitado a lo que se refiere por cliente y empresa hacia delante de la lectura, es importante y a la vez interesante tomar en cuenta cómo es que una persona se convierte en cliente de una empresa, es decir qué proceso pasa este individuo para finalmente adquirir un bien o servicio ofrecido en el mercado y cómo es que la empresa influye en el proceso de esta toma de decisión y en cómo un cliente vuelve a adquirir su producto.

Kotler, Bowen, Makens, García y Flores (2011) exponen que el proceso por el cual pasa un individuo para tomar una decisión de compra se inicia con el reconocimiento de la necesidad, luego con la búsqueda de información, la evaluación de alternativas, la decisión de compra y finalmente el comportamiento posterior a la compra. Para un mejor entendimiento de este proceso que atraviesa un individuo se aprecia en la Figura 1.

El proceso de compra expuesto por Kotler et al. (2011) resalta que el interés de la empresa no acaba cuando el individuo efectúa la compra, sino que esta debe de estar interesada desde el inicio, es decir desde el reconocimiento de la necesidad, hasta el comportamiento de que tiene el individuo luego de obtener el servicio o bien, pues este es el punto de partida donde entrará a tallar los concepto de fidelización y retención del cliente; ya que lo óptimo será que la empresa produzca que el individuo salte algunos pasos de este proceso e ir directamente a efectuar la compra del bien o servicio.

Como se indicó, el objetivo de toda empresa es que todos los clientes omitan ciertas fases del proceso de compra y se dirija directamente a la decisión de compra eligiendo finalmente su marca. Por ello, se explicarán las dos últimas fases del proceso de compra que exponen Kotler et al. (2011), la decisión de compra y el comportamiento post-compra de los clientes:

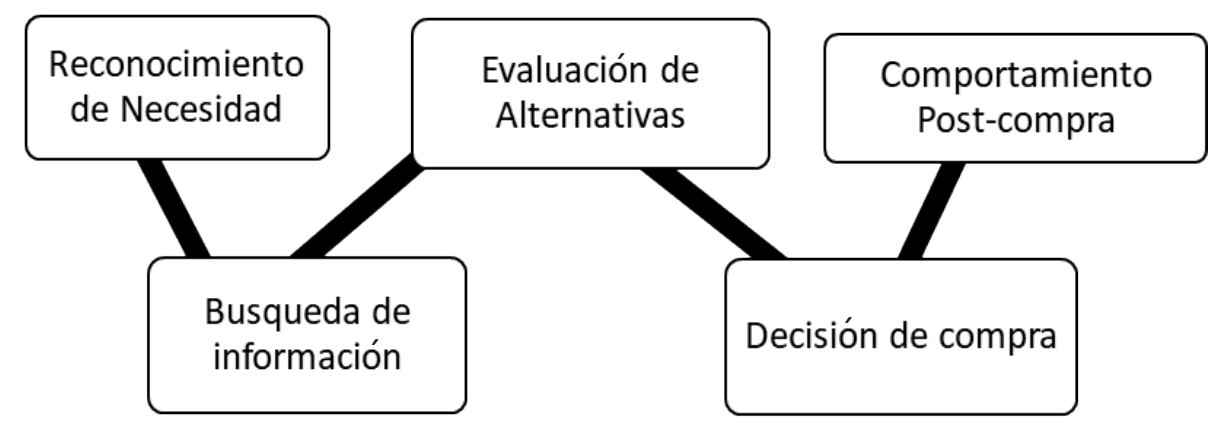

Figura 1. Proceso de compra. Elaboración propia. 
1. Decisión de compra: esta fase es la situación donde el individuo ya reconoció sus necesidades y realizó una exhaustiva evaluación de las diferentes alternativas y finalmente decidió comprar el producto que se sincronizan con dichas necesidades. Sin embargo, la decisión que toma el cliente tiene dos variables que influyen en esta acción, son las actitudes de otras personas y los factores situacionales espontáneos:

a. La variable de las actitudes de otras personas se refiere a la influencia que tiene la opinión de otros individuos frente a un producto u otro. Dichas influencias pueden ser positiva, cuando las opiniones de los diferentes clientes son a favor del producto de interés del nuevo consumidor por lo que fortalecerá el sentimiento de compra del bien o servicio, o pueden ser negativas, lo cual tiene un sentido contrario a las opiniones positivas lo que genera inseguridad al momento de decidir la comprar de un nuevo cliente provocando que este cambie de decisión al último momento.

b. La variable situacionales o espontáneos explican cómo eventos imprevistos pueden provocar una cancelación de la decisión del nuevo cliente, puede ser un accidente que le ocurra al propio cliente o simplemente una acción de la competencia que captó al cliente ofreciendo una mejor opción, sin embargo, esta variable puede ser mitigada con una atención de la empresa por su cliente que debe seguir al cliente hasta que efectué la compra.

2. El comportamiento post-compra del cliente: este concepto se refiere que las empresas no deben interesarse por el cliente hasta que efectué una compra, sino que estas deben de estar interesadas por sus clientes para que estos generen opiniones positivas de la empresa y de esta forma genere e incentive la llegada de nuevos individuos interesados por comprar el bien o servicio ofrecido.

Es decir, esta fase puede llegar a ser doblemente importante para las empresas, pues es en esta fase donde se confirma que el bien o servicio ofrecido es efectivo para satisfacer al mercado y también porque si esto es así estará creando un sistema de buenas referencias hacia la empresa, lo que en las mejores situaciones cause una reducción en el costo para captar nuevos clientes pues sus principales promotores para la llegada de nuevos interesados serán sus clientes ya satisfechos.

Dado que la decisión de compra y los factores improvistos a los que están expuestos los clientes están determinados por incentivos o estimulaciones externas, es importante resaltar el papel que cumple la publicidad y cómo esta es un papel importante para lograr la fidelización de los clientes y más aún para lograr la retención de estos.

Por lo que es importante entender el marketing, aunque sería importante profundizar como el marketing puede llegar a impactar en el éxito de una empresa no es el fin de este artículo, como se explicó es un papel importante para entender el concepto de fidelización y retención del cliente, por ello se toma lo que explica Kotler y Lane (2006) quienes mencionan que el marketing radica en identificar y compensar las necesidades que presentan los individuos y la sociedad, pero también se puede decir que el marketing consiste en satisfacer necesidades de forma rentable.

Esta definición aporta la idea que se está tratando de explicar en el presente artículo, pues como explican Kotler y Lane (2006), el marketing es una herramienta importante para poder lograr la fidelización y la retención de los clientes, dado que es más rentable generar un sentimiento de familiaridad con los clientes y crear algún tipo de grupo diferenciado que es una estrategia más potencial, ya que habrá más personas interesadas en obtener el producto solo por querer pertenecer a dicho grupo diferenciado, en lugar de producir publicidad para atraer nuevos clientes y dejar por decir olvidados a los clientes captados.

Continuando con la línea definida sobre el marketing, es importante resaltar la creciente influencia creada por el marketing digital que está superando en el impacto sobre el público, se refiere en comparación de la publicidad tradicional, tal como lo explica Kotler et al. (2011), que 
el uso intensivo de Internet y de las tecnologías ha tenido un drástico impacto sobre los compradores como sobre las empresas, transformado fundamentalmente el concepto que tienen los clientes de comodidad, rapidez, precio, información sobre productos y servicios. Así la influencia de la tecnología en el mercado ha establecido nuevas formas de crear valor para los clientes y construir relaciones con ellos.

El tener delimitado la noción sobre lo que es el cliente y la empresa, el proceso de compra por el que pasa un individuo, tomando en cuenta ciertos factores dentro de la fase de decisión de compra y post-compra, y cómo es que la publicidad influye altamente en cómo lograr que un cliente se vuelva más rentable para la empresa, es importante para tener un mejor entendimiento previo a la delimitación de los conceptos de fidelización y retención del cliente; además de facilitar el entender como el fortalecimiento de estos conceptos dentro de la empresa pueden a ser muy beneficioso para estas.

Por tanto, se iniciará abordando el concepto de fidelización del cliente para luego continuar con el concepto de retención del cliente.

Entonces, ¿qué es la fidelización del cliente? Iniciaré tomando la explicación de Promove Consultoría e Formación SLNE (2012), donde explica que por fidelización se puede entender a toda estrategia dirigida a alcanzar que los clientes mantengan relaciones sólidas con la empresa a lo largo del tiempo y el objetivo de esta estrategia es crear con el cliente un sentimiento positivo hacia el negocio, que sea lo que motive ese impulso de adhesión continuada generando que el individuo mantenga un volumen de compra constante o que este aumente su volumen de consumo.

Por otro lado, Alcaide (2015) refiere que la fidelización del cliente "es un conjunto de condiciones que permiten al cliente sentirse satisfecho con el producto o servicio que requiera y esto lo impulse a volver a adquirirlo" (p. 18).

Según Pérez (2015) reflexiona sobre la fidelización del cliente como:

una herramienta que ha ido evolucionando con el paso del tiempo. Esta fidelización se encuentra dentro del marketing relacional como uno de los principales elementos del mismo dado que implica lograr tener una retención del cliente en forma global, lo cual permite tener una relación duradera con el cliente. Igualmente, precisa tener un enfoque estratégico orientado a profundizar las estrategias que se van a utilizar para poder fidelizar de mejor manera al cliente. Muchas empresas descuidan la fidelización del cliente, ignoran que ahora es más fácil fidelizar a un cliente antes de captar a un nuevo cliente, es por eso que las empresas han tenido que tomar prioridad al marketing relacional ya que es uno de los principales para poder tener una relación a largo plazo con los clientes.

Al respecto Sánchez (2017) refiere lo siguiente:

La fidelización de clientes genera menos gastos en marketing. Un consumidor que ya nos ha comprado, ya conoce nuestra marca y es más probable que vuelva a comprarnos que un consumidor nuevo, $\mathrm{y}$ a su vez, un cliente habitual requiere de menos operaciones en los procesos de venta.

La fidelización de clientes es una forma de asegurar ventas, ya que es más fácil y barato conseguir que un cliente repita comprar, a que un cliente nuevo compre.

La fidelización se entiende cómo una acción dirigida a conseguir que los clientes mantengan relaciones estables y continuadas con la empresa a lo largo del tiempo. El fin es crear con el cliente un sentimiento positivo hacia el negocio, que sea lo que motive ese impulso de adhesión continuada.

La fidelización se puede entender de dos maneras: la primera que el cliente siga comprando a lo largo del tiempo y la segunda en aumentar su volumen de compra.

Con las definiciones expuestas se puede delimitar el concepto de fidelización del cliente con la empresa y se puede mostrar que existe una diferencia con el otro concepto de retención del cliente, sin embargo, a estas definiciones se puede agregar además la influencia 
de las tecnologías pues no solo es la constante visita de un establecimiento, sino, también de la visita constante de las tiendas virtuales de la empresa que realiza el cliente para estar al tanto de las nuevas promociones que le ofrece o los nuevos productos expuestos.

A continuación, se va a exponer cómo es que se puede lograr la fidelización de los clientes y cómo es que estas políticas pueden beneficiar a las empresas.

Promove Consultoría e Formación SLNE (2012), explica que para lograr la fidelización de los clientes dependerá si y solo si de: si el bien o servicio ofrecido cumple con la calidad prometida; si los trabajadores demuestran un alto conocimiento de los bienes o servicios que se ofrecen y muestran respeto a los clientes, asegurándose que siempre sus dudas sean resueltas; si se preocupan por la satisfacción de sus clientes; si actúan espontáneamente con los clientes detectando que tienen algún problema y por último se debe de establecer una relación personal con ellos.

Para ello, es importante que la empresa tenga una política que incentive el adelanto de las necesidades de sus clientes, es decir, que la empresa siempre debe estar preparada para posibles situaciones de dificultad que presenten sus clientes con el fin de poder solucionarlos rápidamente y de esta forma generar un sentimiento de respaldo percibido por el público generando una fidelización con la empresa.

Además, con la influencia de la tecnología en el comportamiento del público causa que las empresas tomen medidas de manera rápida, pues la información al estar de manera virtual es más accesible y para que se genere la fidelización del cliente se deberán aplicar tres acciones importantes sobre el uso de la tecnología:

- Manejo de datos: Una recopilación y organización de datos, luego se realiza un análisis y tomar las medidas correctivas.

- Implantación de programas: una vez identificados los clientes, sus necesidades y deseos, se desarrollan estrategias para lograr la lealtad de estos clientes.
- Retroalimentación: se realizan los contactos con los clientes y se hace un seguimiento de las preferencias y comportamientos a fin de establecer una relación de largo plazo.

Por lo tanto, es importante que las empresas tengan bien estructurados sus estándares con los cual se puedan asegurar que la calidad ofrecida sea la esperada por el mercado, tener la tecnología necesaria con la cual sea fácil el manejo de información que generan los clientes con el fin de poder solucionar posibles fallas y tener políticas claras con las cuales se incentive la actualización de las dos primeras condiciones, todo esto con el objetivo que el cliente siga interesado en consumir el producto nuevamente y lo recomiende, es decir establecer la fidelización del cliente con la empresa.

Luego de aplicar todas estas medidas, expuestas anteriormente, se podrán percibir los beneficios de tener una fuerte fidelización de los clientes con la empresa. Gummesson (2004) explica uno de estos beneficios, es que los clientes leales son menos sensibles al precio; pero esta actitud del cliente fiel no puede generar una conducta abusiva por parte de la empresa ya que el cliente también valora la confianza y el compromiso.

Mejorar la rentabilidad no viene ni por aumentar los ingresos ni por reducir los costos operativos, sino muy por el contrario aumentar la retención de los clientes ya que se estarían minimizando los costes del marketing para captar nuevos clientes y además de mantener un consumo mínimo asegurado por esto clientes leales o fieles.

También la empresa se puede beneficiar por el sentido que al tener satisfecho a sus clientes y generar un vínculo dándole ciertos beneficios, provoca que el público nuevo vea a la empresa como la mejor posicionada dentro del mercado, es decir que la empresa será percibía como la mejor, por tal motivo esto genera mayor atracción de nuevos cliente, los cuales tendrán como primera opción a nuestra empresa y se estaría adquiriendo a la vez clientes fidelizados desde el inicio pues entra con una alta expectativa sobre la calidad del bien o servicio y esta se ve retribuida con las medidas que la empresa aplica con sus clientes, en conclusión de crea un ciclo de buenas opiniones 
sobre la empresa generando mayor interés del mercado por ser parte de su clientela.

Por otro lado, ¿qué es la retención del cliente? Bardakci y Whitelock (2003) opinan que las políticas para lograr la retención del cliente es importante tener en cuenta lo siguiente: adquirir nuevos clientes es una mayor inversión para la empresa que mantener los existentes, el cliente satisfecho volverá a comprar, clientes satisfechos transmitirán su satisfacción a otros tres; o lo opuesto, clientes no satisfechos se lo dirán a nueve. Además, Kotler (2000) agrega que la clave para lograr la retención del cliente está en conseguir la satisfacción, pues los clientes satisfechos permanecen leales, generan opiniones positivas de la empresa, ponen menos interés en la competencia, aportan ideas de mejora a la empresa y cuesta menos servirles que a un cliente nuevo. Por ende, la retención del cliente es el transformar un cliente insatisfecho con el bien o servicio adquirido; en un cliente satisfecho y posteriormente fidelizado, pues siente un respaldo frente a sus dificultades con los productos, pero está en la empresa el aprender de este cliente insatisfecho para mejorar el bien o servicio, es decir, se puede tomar a una queja como una sugerencia de mejora y que sea continua.

Entonces, el concepto de generar la retención de clientes no solo incide en la existencia previa de una actitud favorable hacia la empresa, es decir no surge solamente de un cliente fidelizado, sino el concepto únicamente se trata de impedir que los clientes dejen de comprar lo producido por la empresa mediante determinadas acciones que permitan su retención.

Guadarrama y Rosales (2015) refieren que:

la clave para la retención del cliente es la satisfacción, pues los clientes satisfechos permanecen leales más tiempo, hablan favorablemente de la empresa, ponen menos interés en la competencia, son menos sensibles al precio, ofrecen ideas de mejora a la empresa y cuesta menos servirles que a un cliente nuevo. (p. 328)

Por lo tanto, por lo explicado anteriormente el retener a los clientes si genera un impacto positivo en la empresa, pues como lo explica Promove Consultoría E Formación SLNE (2012), ya que obtener un nuevo cliente puede costar 5 veces más de lo que costaría mantener un cliente que deja de serlo por problemas surgidos en la gestión de su relación comercial y por tal razón es vital conocer las opiniones, las quejas que se dan a la empresa y contrastarlos con los servicios que el negocio les ofrece.

Es así como el concepto de retención del cliente insatisfecho y la existencia de quejas o descontentos beneficia a la empresa, es decir, un grado moderado de insatisfacción de los clientes favorece una evolución sostenible de los bienes o servicios que se ofrezcan. Esto es así porque con estas insatisfacciones se pueden conocer ciertas necesidades no cubiertas por los ofrecido y pueden resultar reflexiones para el mejoramiento del bien o servicio, también al lograr solucionar la situación de insatisfacción por parte del cliente, se transforma ese mal rato como una grata experiencia con lo cual genera una doble opinión positiva que será reciba por nuevos clientes, mejorando así las referencias que tiene el mercado de la empresa.

Finalmente, es interesante tener métodos para medir los grados de fidelización y también sobre la retención de los clientes. Sobre la fidelización, se puede tomar el volumen de clientes afiliados a tarjetas de beneficios, montos de cupones ofrecidos y consumidos o la cantidad de puntos canjeados, todo esto con el fin de tener un monitoreo del grado de fidelización que se tiene de los clientes de la empresa.

Por la parte de la retención, Weinstein (2002) formula un modelo de retención/valor para el cliente que relaciona la satisfacción y el desarrollo del negocio. En el cual explica que dando una mayor importancia al cliente mediante una mezcla de calidad, servicio y precios se logrará exceder sus expectativas, o explicaría el aumento del incentivo por volver a consumir los bienes o servicios ofrecidos por la empresa. Por lo tanto, el grado de satisfacción lleva a una mayor lealtad y a un incremento de la rentabilidad del negocio y finalmente de la retención del cliente.

En suma, la fidelización y retención del cliente son conceptos muy importantes para poder asegurar que el desarrollo de la empresa sea sostenible y estable, esto sería gracias a que se asegura un público fiel que consumirá las producciones nuevas que se genere en la 
empresa. También esta idea se fundamenta en los beneficios que genera el tener un grupo leal de clientes, pues se reducen los costos de publicidad, ya que la principal fuente de publicidad serán las opiniones que generen los participantes de este grupo fiel de clientes.

\section{CONCLUSIONES}

Se entiende entonces por cliente como a la persona o empresa que genera un transacción o compromiso de compra futura con una entidad para adquirir un bien o servicio, siendo esta transacción de manera física o virtual. También se entiende por empresa como una entidad que utiliza el capital, materiales, talento humano y la tecnología para generar un producto o servicio que se pueda ofrecer dentro de un mercado determinado, además estas organizaciones buscan maximizar sus beneficios y en busca de ellos este artículo propone que al reforzar las políticas sobre la fidelización y retención de los clientes ya que se estarán reduciendo los costes de publicidad obteniendo doble beneficio.

Como apoyo a lo explicado sobre la actitud maximizadora de las empresas se explicó cómo es que la publicidad es una herramienta útil para ello, pues es más rentable focalizar la publicidad sobre el grupo de clientes que tiene la empresa ya que de esa forma estos clientes serán los que impulsen la marca con sus opiniones positivas de lo que ofrece la empresa y también se crea grupo diferenciado ante los demás, lo que a su vez provoca el interés de un nuevo público que deseará pertenecer a aquel grupo con lo cual consumirán lo producido por la marca.

Dicho lo anterior, se define la fidelización del cliente como algo más que una relación duradera de un cliente con una empresa, se puede decir que es una sensación de afinidad o adhesión a los productos o servicios que ofrece una empresa, esto también incluye el tener al cliente pendiente de las distintas redes de comunicación que tenga la empresa.

Lo que se busca es generar un gran beneficio a la empresa, pues un cliente leal significará un nivel de consumo de los bienes o servicios constantes o el aumento periódico de estos, además de que un cliente fiel es el mejor medio de publicidad para cualquier negocio, pues como se explicó en el proceso de compra, específicamente en la decisión de compra, la conducta de las personas influyen mucho en esta fase por lo que a mayor cantidad de clientes fieles mayor cantidad de individuos incentivados a consumir los bienes o servicios ofrecidos por la empresa en lugar de ir por la competencia.

Por otro lado, se define la retención del cliente como la transformación de un cliente insatisfecho con el bien o servicio adquirido; en un cliente satisfecho y posteriormente fidelizado, pues siente un respaldo frente a sus dificultades con los productos. Esto no implica la existencia previa de una actitud especialmente favorable hacia la empresa, sino el concepto únicamente se trata de impedir que los clientes dejen de comprar lo producido por la empresa mediante determinadas acciones que permitan su retención.

De este modo el concepto de retención del cliente insatisfecho y la existencia de quejas o descontentos se puede tomar como una oportunidad de beneficio para la empresa, ya que con estas insatisfacciones se pueden conocer ciertas necesidades no cubiertas por los ofrecido y lo que trae como resultado reflexiones para el mejoramiento del bien o servicio y así las referencias que tiene el mercado de la empresa.

En tal sentido, queda claro que la existente diferencia entre los conceptos de fidelización del cliente y la retención del cliente. Esta diferencia parte desde cómo está la relación del cliente con la empresa luego de haber consumido lo ofrecido por ella, si esta relación es de satisfacción y la empresa continua la comunicación y alimentando este sentimiento causará la fidelidad del cliente con la empresa; lo opuesto ocurre cuando el cliente está insatisfecho con lo adquirido de la empresa, con lo cual ahí entra a tallar las políticas de retención que tengan las empresas con el fin de transformar el sentimiento negativo en uno positivo y se convierta en un cliente fiel, pues reconoce que ante cualquier dificultad con el producto la empresa estará al tanto para solucionarlo y también esto genera un mayor reconcomiendo dentro del mercado.

Finalmente, luego de delimitar los conceptos de fidelización del cliente y la retención del cliente, se enfatiza la importancia de estos dos 
conceptos y la importancia que deben de darle las empresas para lograr mayores retornos, solo es necesario redirigir las políticas de relación que deben tener los colaboradores con los clientes para producir un sentimiento de cercanía.

\section{REFERENCIAS BIBLIOGRÁFICAS}

Alcaide, J. (2015). Fidelización de clientes. Madrid: ESIC. Recuperado el 19 de marzo de 2016, de https://books.google.com.ec/ books? id=GYA08Sbe63 cC\&printsec = frontcover \&dq=estrategia $+\mathrm{de}+$ marketing2010\&hl=es-19\&sa=X\&redir_esc=y\#v=onepage\&q\&f=false

Bardakci A. y Whitelock J. (2003). Mass-customization in marketing: the consumer perspective. Journal of consumer marketing, 5(20), 463-479.

Gummesson E. (2004). Return on Relationships (ROR): the value of relationship marketing and CRM in business-to-business contexts. The Journal of Business \& Industrial Marketing, 2(19), 136-148.

Guadarrama, E. y Rosales, E. (2015) Marketing Relacional: Valor, Satisfacción, Lealtad y Retención Del Cliente. Análisis y Reflexión Teórica. Ciencia y Sociedad, vol. 40, núm. 2, 2015, pp. 307340. Instituto Tecnológico de Santo Domingo. Santo Domingo, República Dominicana
Kotler P. (2000). Marketing Management. Estados Unidos, New Jersey: Prentice Hall.

Kotler P., Bowen J., Makens J., García J. y Flores J. (2011). Marketing Turístico. España, Madrid: Pearson Educación S.A.

Kotler P. y Lane K. (2006). Dirección del Marketing. México: Pearson Educación de México.

Pérez, M. (2015). ¿Qué son los programas de fidelización y por qué debes apostar por ellos? Obtenido de https://blog.hubspot.es/marketing/ que-son-los-programasde-fidelizacion

Promove Consultoría E Formación SLNE (2012). Atraer y fidelizar clientes, Cuadernos Prácticos, Gestión Empresarial. España, Galicia: C.E.E.J. Galicia S.A.

Sánchez, S. (2017). Negocios y Empresas. Obtenido de: https://www.puromarketing. com/14/28784/fidelizacion-clientes.html

Real Academia Española (s.f.). Diccionario de la lengua española. Recuperado de https://dle.rae.es/ cliente? $\mathrm{m}=$ form

Weinstein A. (2002). Customer-Specific Strategies. Customer retention: Ausage Segmentation and Customer Value Approach. Journal of Targeting, Measurement and analysis for Marketing, 3(10), 259-268. 
